40-1| 2015

Enjeux contemporains en études irlandaises - In Memoriam Paul Brennan

\title{
Les rapports entre l'État irlandais et ses citoyens après la crise : quelles orientations ?
}

\section{Julien Guillaumond}

\section{(2) OpenEdition \\ Journals}

Édition électronique

URL : http://journals.openedition.org/etudesirlandaises/4459

DOI : 10.4000/etudesirlandaises.4459

ISSN : 2259-8863

\section{Éditeur}

Presses universitaires de Rennes

Édition imprimée

Date de publication : 30 juin 2015

Pagination : 71-87

ISBN : 978-2-7535-4082-8

ISSN : 0183-973X

Référence électronique

Julien Guillaumond, « Les rapports entre l'État irlandais et ses citoyens après la crise : quelles orientations? », Études irlandaises [En ligne], 40-1 | 2015, mis en ligne le 30 juin 2017, consulté le 19 avril 2019. URL : http://journals.openedition.org/etudesirlandaises/4459 ; DOI : 10.4000/ etudesirlandaises. 4459 


\title{
Les rapports entre l'État irlandais et ses citoyens après la crise : quelles orientations ?
}

\author{
Julien Guillaumond \\ Université Blaise-Pascal Clermont-Ferrand 2
}

\section{Résumé}

Jadis perçu comme modèle économique, l'Irlande fut l'un des pays les plus touchés par la crise financière de 2008. Les transformations que la société irlandaise a connues au cours des deux dernières décennies, couplées à l'insécurité économique, politique mais aussi sociale générée par la crise, ont-elles conduit à une reconfiguration des rapports entre l'État et ses citoyens? Entre émigration et demandes pour davantage de transparence et de participation citoyenne par le biais des nouvelles technologies, quelles grandes tendances se dessinent?

Mots clés : débat et sphère publics, Etat irlandais (République d'Irlande), Irlande - politiques économiques et sociales

\section{Abstract}

Once seen as a successful economic model, Ireland was among the countries hardest hit by the financial crisis. Have the transformations undergone by Irish society over the last two decades, together with economic, political and social insecurity caused by the crisis, led to a re-configuration of the relationships between the State and its citizens? Considering demographic exile and demands for more transparency and citizens' participation through the use of new technologies, is it possible to identify major trends?

Keywords: public debates, Irish State (Republic of Ireland), Ireland-socio-economic policies

La crise financière mondiale qui a frappé l'Irlande et provoqué la mise sous tutelle du pays fin 2010 pour garantir sa solvabilité, dans un contexte déjà marqué par la chute des économies grecque, portugaise et espagnole, a donné lieu à de nombreuses interrogations, tant sur les raisons de l'effondrement du modèle économique irlandais que sur le rôle des acteurs économiques et politiques. L'éditorial de l'Irish Times du 18 novembre 2010 donnait alors le ton, soulignant le traumatisme que pouvait représenter l'arrivée d'experts de la troïka venus au chevet de l'économie irlandaise :

1. Reposant sur une étude de travaux universitaires récents, de sources primaires et de la littérature grise, cet article s'inscrit dans une démarche plus exploratoire que théorique sur la reconfiguration des rapports entre État et citoyens en Irlande au $\mathrm{XxI}^{\mathrm{e}}$ siècle. 
La véritable ignominie au regard de notre situation actuelle, ce n'est pas que l'on nous ait pris notre souveraineté, c'est plutôt que nous l'ayons perdu tout seuls. [...] L'incompétence des gouvernements que nous avons élus a profondément compromis notre capacité à prendre nos propres décisions ${ }^{2}$.

Péniblement acquise dans la première moitié $\mathrm{du} \mathrm{xx}^{\mathrm{e}}$ siècle, la souveraineté économique et politique de l'Irlande s'était envolée. Des instances internationales extérieures - Commission européenne, Banque centrale européenne et Fonds monétaire international - étaient dorénavant en charge des affaires du pays. Le 19 novembre, l'inauguration du nouveau terminal aéroportuaire de Dublin par le Taoiseach, Brian Cowen, offrait un contraste saisissant révélant une certaine insouciance, quasi surréaliste, voire un déni complet de la situation. Soulignant la dimension visionnaire du bâtiment, B. Cowen expliquait qu'il

serait extrêmement réducteur de considérer [le Terminal 2] au regard du ralentissement économique actuel. En investissant avec prudence dans la modernisation des infrastructures, nous jetons les bases d'une croissance et d'une prospérité à venir, pas uniquement pour l'aéroport de Dublin, mais aussi pour l'économie irlandaise dans son ensemble ${ }^{3}$.

Rétrospectivement, l'inauguration de cette infrastructure peut être considérée comme le point de basculement où se côtoient vision du passé et vision de l'avenir. Si elle semble représenter le point culminant de deux décennies d'euphorie économique, elle peut aussi être l'indicateur malheureux d'un retour de l'émigration puisqu'à mesure que croissent les capacités d'accueil de l'aéroport, les capacités de départ vers l'étranger qu'offre ce nouveau terminal augmentent également.

Si la question de la perte de la souveraineté irlandaise semblait primordiale dans de nombreux articles de journaux ${ }^{4}$, elle ne représentait qu'une des dimensions du problème. Une seconde question prenait de l'ampleur : la chute de confiance dans les autorités du pays, coincées entre insouciance et refus de voir la situation en face. Partant d'une évaluation des perceptions irlandaises sur

2. "The true ignominy of our current situation is not that our sovereignty has been taken away from us, it is that we ourselves have squandered it. [...] It is the incompetence of the governments we ourselves elected that has so deeply compromised our capacity to make our own decisions. "Irish Times, "Was it for all this?", 18 novembre 2010. La répétition de« we ourselves " fait écho aux aspirations nationalistes irlandaises du début du $\mathrm{xx}^{\mathrm{e}}$ siècle inscrites dans le "nous-mêmes " du Sinn Féin.

3. "To view it [Terminal 2] through the prism of the current downturn would be short-sighted in the extreme. By investing prudently in improved facilities, we are laying the foundations for future growth and prosperity; not just for Dublin Airport, but also for the wider Irish economy [...] ". Dublin Airport, "Taoiseach Opens Terminal 2 ", 19 novembre 2010, [http://www.dublinairport.com/gns/at-the-airport/latest-news/10-11-19/Taoiseach_Opens_Terminal_2.aspx], page consultée le 5 décembre 2014. Nous soulignons.

4. Voir par exemple l'article de Paul O’Brien, Ann Cahill et Mary Regan, "Proclamation of Dependence”, Irish Examiner, 24 décembre 2010, accompagné d'une photo des experts de la troïka débarquant à Dublin. 
la gestion de la crise par le gouvernement en place et de réflexions issues des travaux de l'économiste américain Albert $\mathrm{O}$. Hirschman sur le comportement des acteurs, cet article s'interroge sur les tendances à l'œuvre dans la société irlandaise au regard des notions de transparence et de responsabilité à l'aune des possibilités offertes par les technologies de la communication. L'étude d'une initiative citoyenne ainsi que l'examen de la position du gouvernement irlandais sur la notion de transparence permettent d'apporter quelques premiers éléments de réponse.

\section{Perte de confiance et malaise populaire}

La seconde quinzaine de novembre 2010, consacrée en partie aux discussions sur le plan de sauvetage de l'économie irlandaise, semble dès lors cristalliser les colères et le manque de confiance vis-à-vis des élites, mais aussi le sentiment d'abattement et de malaise qui règnent dans la société irlandaise et dont la presse irlandaise se fait l'écho. Rappelons ici quelques-uns des faits saillants : une croissance économique en berne (-2,2 \% en $2008,-6,4 \%$ en $2009,-1,1 \%$ en 2010) ${ }^{5}$; un taux de chômage qui explose de 5,6 à 12 \% entre 2008 et 2009; un État irlandais qui a garanti les dépôts et les prêts octroyés par les établissements bancaires; la nationalisation de l'Anglo-Irish Bank en janvier 2009; le lancement de trois commissions d'enquête en 2010 sur la crise bancaire'.

La fête était finie, rappelait Lisa O'Carroll, correspondante du Guardian en Irlande ${ }^{7}$. Si la question de la souveraineté était importante pour les Irlandais, les entretiens qu'elle avait réalisés suggéraient toutefois que la présence des experts du FMI était ressentie à la fois comme la garantie d'une certaine stabilité à venir, mais aussi comme le moyen de palier un manque de confiance dans un gouvernement accusé de dissimuler la vérité ${ }^{8}$. Joseph O’Connor proposait un constat semblable tout en dénonçant l'aveuglement des élites dirigeantes qui auraient abusé du système, et la société irlandaise dans son ensemble.

Après avoir vécu la dernière décennie dans une brume enivrante d'autosatisfaction vis-à-vis de notre réussite économique, nous faisons face dorénavant à la réalité que tout ceci n'était qu'illusion. Des politiciens incompétents, des banquiers avides et des spéculateurs immobiliers

\footnotetext{
5. D’après Eurostat, base de données en ligne, [http://epp.eurostat.ec.europa.eu].

6. Gary Murphy, «The Background to the Election ", in Michael Gallagher et Michael Marsh, (éd.), How Ireland Voted 2011. The Full Story of Ireland's Earthquake Election, Basingstoke, Palgrave Macmillan, 2011, p. 17.

7. Lisa O'Carroll, "Ireland has mixed feelings over multi-billion euro IMF bailout", The Guardian, 18 novembre 2010.

8. Ibid.
} 
ont provoqué l'écroulement des certitudes sur lesquelles, jusqu'à présent, était fondée notre perception de nous même?

Son réquisitoire se poursuivait en ces termes :

Les gens en sont venus dans l'ensemble à ne plus avoir confiance dans le gouvernement [conduit par Brian Cowen] et, j'ai horreur d'employer le mot, à exécrer ce même gouvernement. [...] J'étais jeune dans les années 80 . Je sais ce que récession veut dire. Mais je ne me souviens pas de cette colère explosive dorénavant présente, ce sentiment de trahison et d'injustice. [...] Et cette colère s'ancre dans un contexte plus large, parfois passé sous silence : le fait que tant de personnes soient coupables, pas seulement quelques-unes. La culpabilité n'est pas seulement l'apanage de promoteurs immobiliers et de leurs amis banquiers que l'on peut corrompre, ni ne dépend seulement d'une succession de gouvernements irlandais incroyablement imprudents; elle provient plutôt de notre société, et de ses composantes les plus officielles. Voilà ce qui rend l'Irlande si profondément mal à l'aise ${ }^{10}$.

Bien qu'incertaine, la situation dans laquelle se trouvait le pays n'en restait pas moins floue. Interrogé sur ce point, l'historien irlandais Diarmaid Ferriter déplorait l'absence d'explications sur la situation et le silence manifeste du gouvernement.

Nous pouvons effectivement parler d'une perte de souveraineté, mais il ne s'agit que de l'aboutissement de deux années d'une absence totale d'orientations données par les dirigeants [...]. Et il est tout aussi extraordinaire de remarquer que compte tenu de l'ampleur de la crise, nous n'avons pas encore eu une déclaration cohérente de la part du gouvernement expliquant aux citoyens ce qui se passe ${ }^{11}$.

9. "Having spent the last decade in a fog of intoxicating self-congratulation for our economic success, we now face the reality that it was illusory. Inept politicians, greedy bankers and property speculators have wrecked the certainties on which our recent notions of ourselves were founded". Joseph O'Connor, "Irish people feel frightened, alone and unled", The Guardian, 18 novembre 2010.

10. "His [Brian Cowen's] administration came to be widely mistrusted and - I hate to use the word-loathed. [...] I was young in the 1980s. I know what a recession is. But I cannot remember the boiling anger that now exists here, the sense of betrayal and injustice. [...] And there is a wider, if sometimes unacknowledged, context for the anger. It is that we know the culpability belongs to so many, not to few: not only to the developers and their venal friends in the banks, not only to a succession of appallingly reckless Irish governments, but to our very society itself, and its very official element. This is what is making Ireland so deeply uneasy. "J. O'Connor, "Irish people feel frightened, alone and unled", op. cit.

11. "I know we can talk about the loss of sovereignty, but this is the culmination of two years of complete lack of direction of leadership [...]. And it is extraordinary that, given the scale of the crisis, that we still have not had a coherent statement from the government to the citizens to tell us what is going on. " Lisa O'Carroll, "Ireland is losing the propaganda war ", The Guardian, 19 novembre 2010. 
Si un communiqué du Premier ministre fut finalement publié quelques jours plus tard, le 21 novembre 2010, expliquant l'intervention de la trö̈ka, il n'en demeure pas moins que ce temps de latence auquel s'ajoutent les différents démentis que les Irlandais ont pu entendre de la part de plusieurs ministres jusqu'à celui des Finances, Brian Lenihan, au cours de la semaine précédente $^{12}$, conduisent au manque de confiance en l'équipe gouvernementale. Pour d'autres observateurs comme Stephen Collins, le flou qui régnait quant à la situation réelle du pays n'était pas tant lié à la volonté du gouvernement de nuire aux citoyens qu'à son incompétence ${ }^{13}$. Dans un contexte de méfiance visà-vis du gouvernement, de malaise et d'incertitudes face à l'avenir, un changement de gouvernement apparaissait comme la seule alternative possible. C'est ce que proposait John Gormley, dirigeant des Verts et ministre de l'Environnement dans la coalition au pouvoir, en appelant à un vote sanction et à l'élection prochaine d'un nouveau gouvernement, soulignant que " [la] semaine qui s'est écoulée fut l'une des plus traumatisantes pour l'électorat irlandais » et que " [les] gens se sentent trompés et trahis ${ }^{14}$ ". Lors d'un discours aux Irish Book Awards, la Présidente irlandaise, Mary McAleese, pouvait alors difficilement occulter ce malaise ressenti par la population ainsi que la situation dans laquelle se trouvait le pays :

Tout en restant dans le cadre dicté par ma fonction, je veux souligner la consternation et la détresse bien réelles de nos concitoyens dans tout le pays qui sont inquiets pour leur avenir. [...] Pour faire face aux difficultés actuelles, sincérité, responsabilité et débats sont nécessaires afin de s'assurer que les graves erreurs du passé ne se reproduiront plus ${ }^{15}$.

\section{La recherche de responsables}

$\mathrm{Si}$, comme rappelé plus haut, ces sentiments de malaise, de perte de confiance dans le gouvernement (sa cote de confiance ayant littéralement chuté entre 2008

12. G. Murphy, "The Background to the Election ", in M. Gallagher et M. Marsh (éd.), How Ireland Voted 2011, op. cit., p. 25.

13. Stephen Collins, "Early election needed to draw line under ignominy", The Irish Times, 22 novembre 2010.

14. "The past week has been a traumatic one for the Irish electorate. People feel misled and betrayed. " Irish Independent, "Government in crisis as Green Party calls for general election", 22 novembre 2010. La tenue prochaine d'élections législatives fut annoncée peu après. Celles-ci eurent lieu le 25 février 2011.

15. "While fully respecting the nature of my role, I want to acknowledge the understandable distress and dismay being experienced by people all around the country who feel fearful about their future. [...]. In facing up to the present difficulties, there needs to be candour, accountability and debate to ensure that the grave failures of the past are never repeated. " "Remarks by President McAleese at the Irish Book Awards", Mansion House, 25 novembre 2010, [http://www.president.ie/index.php?section $=5$ ospeech=895 rlang=eng], page consultée le 23 décembre 2010. 
et 2009$)^{16}$, voire de trahison pour certains, sont à considérer à la lumière des événements qui, depuis 2008, ont précédé ces semaines fatidiques de novembre, ils sont pour partie également liés aux débats relatifs aux choix de société en Irlande depuis l'avènement du Tigre celtique. À ces interrogations sur l'évolution de la société irlandaise dans une économie mondialisée, sur les rapports qu'entretiennent les citoyens et l'État et sur la place même de l'État irlandais et de ces politiques économiques et sociales, un vaste ensemble d'ouvrages a cherché à proposer des éléments d'analyse, constituant dès lors une toile de fond utile pour l'analyse $\mathrm{e}^{17}$. Selon la période et la finalité recherchée, les auteurs, journalistes ou universitaires soulignent tantôt le rôle des acteurs et des institutions, du gouvernement, de la fonction publique mais aussi des établissements bancaires, responsables d'avoir engendré la crise et/ou de n'avoir pas su la prévoir et/ou l'anticiper, tantôt le modèle économique et social choisi. Certains ouvrages dénoncent ainsi l'aveuglement des élites dirigeantes, les liens entre monde politique et monde des affaires ou la vénalité des banquiers. Figurant en tête des ventes pendant un temps, ces livres rencontrent un grand succès auprès du public ${ }^{18}$.

D'autres travaux privilégient une approche plus globale. Pour Peadar Kirby et Mary P. Murphy, les raisons de la crise sont notamment à chercher dans les faiblesses du système politique irlandais : un État centralisé où le pouvoir exécutif a l'ascendant sur le Parlement, une culture politique marquée par un fort localisme et l'absence d'une culture de la responsabilité dans l'administration ${ }^{19}$. Niamh Hardiman relève également cette variable politique, insistant notamment sur le déséquilibre de pouvoir entre l'exécutif et le législatif. Si d'autres instances de supervision et/ou de contrôle existent bel et bien, il n'en reste pas moins que celles-ci s'insèrent dans un schéma structurel éclaté qui a pour effet la dispersion de la notion de responsabilitée ${ }^{20}$, y compris par rapport à la crise ${ }^{21}$. Ainsi, si l'adoption d'une stratégie de cooptation des divers intérêts par les gouvernements irlandais successifs pour aboutir aux accords tripartites (État, employeurs et syndicats)

16. Niamh Hardiman, "The Impact of the Crisis on the Irish Political System ", Geary Institute, University College Dublin, document de travail, novembre 2009, [http://ideas.repec.org/p/ucd/wpaper/200932.html], p. 3-4.

17. L'on trouvera ici une liste non exhaustive d'ouvrages, un travail de recensement étant en cours. En complément, l'on pourra se reporter à l'étude critique de Shaun McDaid, "The Crisis in Ireland: where did it all go wrong? ", Political Studies Review, 2014, [http://dx.doi.org/10.1111/1478-9302.12056], page consultée le 30 juin 2014.

18. Shane Ross et Nick Webb, Wasters, Dublin, Penguin Ireland, 2010. Shane Ross, The Bankers. How the Banks Brought Ireland to its Knees, Dublin, Penguin Ireland, 2009. Simon Carswell, Anglo Republic: Inside the bank that broke Ireland, Dublin, Penguin Ireland, 2011. Louise McBride, "Tale of how Celtic Tiger was wasted tops 2010 charts", Irish Independent, 9 janvier 2011.

19. Peadar Kirby et Mary P. Murphy, Towards a Second Republic. Irish Politics after the Celtic Tiger, Londres, Pluto Press, 2011.

20. Niamh Hardiman, "Governance and Accountability: the Limits of New Institutional Remedies ", in Niamh Hardiman, (éd.), Irish Governance in Crisis, Manchester, Manchester University Press, 2012, p. 23-42.

21. Jonathan Westrup, "Regulatory Governance ", in N. Hardiman, Irish Governance in Crisis, op. cit., p. 64-82. 
de partenariat social a effectivement permis, par le passé, de réduire les conflits potentiels tout en forçant à repenser collectivement les problèmes de société, elle a aussi conduit à une certaine homogénéisation des idées ${ }^{22}$.

Dans un pays marqué par une succession de scandales financiers mais aussi religieux, l'ouvrage d'Elaine Byrne constitue un élément central dans la dénonciation du système politique irlandais. La mutation des valeurs, tant au niveau sociétal que politique, peut en partie, selon elle, expliquer l'état de la corruption en Irlande. Dans le même temps, la succession de commissions d'enquête dans les années 1990-2000 sur les affaires politico-économiques mêlant investisseurs privés, fonctionnaires et figures politiques de premier plan $^{23}$, fournit un élément supplémentaire à une société irlandaise méfiante vis-à-vis d'une classe politique trop prête à privilégier ses intérêts par rapport à ceux du pays ${ }^{24}$.

E. Byrne prolonge ainsi le travail engagé auparavant par Muiris MacCarthaigh qui rappelait également le faible pouvoir du Dáil comme instance de contrôle et de surveillance des actions du gouvernement, tout en montrant l'ensemble des dispositifs qui avaient été mis en place pour renforcer le processus démocratique $^{25}$. Le politologue Peter Mair optait pour une position plus critique, rendant les Irlandais responsables de la crise, soulignant leur distance et leur méfiance visà-vis de l'État, leur faible engagement quant aux grandes orientations de société et, paradoxalement, leur préférence pour les questions locales, laissant la gestion des affaires de l'État aux intérêts particuliers, comme l'Eglise mais aussi le secteur financier ${ }^{26}$.

Les lignes précédentes méritent d'être nuancées au regard des responsabilités. En effet, pour certains chercheurs, il semblerait que la recherche des responsables de la situation économique et sociale du pays se soit lentement déplacée vers d'autres catégories que celles identifiées précédemment. Sous l'influence des politiques, des milieux d'affaires et des médias grand public, cette recherche d'explication de la crise a évolué, passant d'un «nous » collectivement coupables, partageant cette responsabilité liée au mythe de l'Irlandais propriétaire, vers d'autres catégories de la population, nettement plus vulnérables : employés du

22. Séan McGraw, "Adaptative Governance: the Art of Party Politics in Contemporary Ireland », in N. Hardiman, Irish Governance in Crisis, op. cit., p. 43-63.

23. Elaine Byrne, Political Corruption in Ireland, 1922-2010. A Crooked Harp?, Manchester, Manchester University Press, 2012.

24. Cela se vérifie avec la création de NAMA (National Assets Management Agency), agence en charge depuis décembre 2009 des emprunts dits toxiques, très rapidement considérée comme l'agence gouvernementale privilégiant les intérêts des banques au détriment de ceux des Irlandais. G. Murphy, "The Background to the Election ", op. cit., p. 21.

25. Muiris MacCarthaigh, Accountability in Irish Parliamentary Politics, Dublin, Institute of Public Administration, 2005.

26. Peter Mair, "We Need a Sense of Ownership of Our State ", in Joe Mulholland, (éd.), Transforming Ireland, 2011-2016, Dublin, The Liffey Press, 2011, p. 38-44. 
secteur public, destinataires des aides sociales et immigrés ${ }^{27}$. Toutefois, en dépit d'une répartition diffuse des responsabilités, une constante demeure au regard des diverses explications de la situation de l'Irlande comme l'ont résumé Eoin O’Malley et Muiris MacCarthaigh.

Si la crise est authentiquement irlandaise, quels sont les facteurs responsables? Nous pouvons souligner des politiques décevantes, un manque de contrôle et de clairvoyance, des faiblesses dans la planification ainsi que des prévisions optimistes qui s'approchaient plus de douces illusions que d'attentes raisonnables sur ce qui pourrait vraiment advenir. Certains soulignent l'incompétence et même la malhonnêteté de nos élus. D'autres fustigent le "système ", arguant que le système politique récompense les personnes qui raisonnent à court terme, qui veillent aux intérêts particuliers et se concentrent presque exclusivement sur les questions locales au détriment des questions nationales. Le système bureaucratique [...] est accusé de faire preuve de trop d'égards pour les intérêts des politiques et des milieux d'affaires, et de ne pas réussir à protéger les intérêts à long terme de l'État ${ }^{28}$.

L'ampleur des critiques vis-à-vis du système politique et économique irlandais ne constitue nullement un cas particulier. De nombreuses sociétés en Europe se sont aussi interrogées autant sur la capacité de leurs dirigeants à les faire sortir de la crise que sur les raisons pour lesquelles cette crise n’avait pu être anticipée. Dans le même temps, leurs citoyens concevaient une méfiance encore plus grande visà-vis des institutions financières et de leurs propres institutions politiques comme ont pu le montrer, par exemple, les études Eurobaromètre sur la confiance dans les gouvernements et les Parlements nationaux. Il est vrai qu'en Irlande, la «manifestation d'un échec systémique de la gouvernance $[. .$.$] a revêtu un caractère parti-$ culier [...] dans la mesure où l'on s'est interrogé à de nombreuses reprises sur le bien-fondé des liens entre politiques, régulateurs, banquiers et promoteurs immobiliers $^{29}$ ".

27. Micheal O’Flynn, Lee F. Monaghan et Martin J. Power, «Scapegoating During a Time of Crisis. A Critique of Post-Celtic Tiger Ireland ", document consulté le 20 juin 2014, [http://ulir.ul.ie/handle/10344/3856, p. 11].

28. "If the crisis is largely home grown, however, what then is to blame? We can point to poor policies, a lack of oversight and foresight, failures in planning and optimistic forecasting that had more to do with wishful thinking than any rational expectation of what would actually happen. Some commentators point to our elected representatives, claiming ineptitude and even dishonesty. Others castigate 'the system', alleging that the political system rewards those who think in the short term, who look after vested interests and concentrate almost exclusively on local rather than national concerns. The bureaucratic system [...] stands accused of being too deferential to political and business interests and failing to protect the long-term interests of the state. " Eoin O'Malley et Muiris MacCarthaigh, Governing Ireland: From Cabinet Government to Delegated Governance, Dublin, Institute of Public Administration, 2011, p. 1-2.

29. "The evidence of systemic failure in relation to governance is echoed in other countries but has taken a particular form in Ireland where troubling questions have been raised about the propriety of the relationships between politicians, 


\section{Exit or Voice : le dilemme irlandais entre émigration et prise de parole}

Dans un contexte marqué par un profond désenchantement à l'égard de la classe politique et des institutions démocratiques, d'une distance vis-à-vis des partis politiques et d'une remise en cause de la participation citoyenne $e^{30}$, peut-on identifier dans l'Irlande d'après crise de grandes tendances sur les relations entre citoyens et État? Un an avant l'événement traumatisant de novembre 2010, N. Hardiman indiquait que "la manifestation la plus évidente de nos problèmes politiques est la chute soudaine et tragique de la confiance citoyenne dans le gouvernement ${ }^{31} »$. Les sondages au sortir des urnes en février 2011 validaient effectivement ce constat. Pour les électeurs irlandais, la crise économique et leur colère vis-à-vis du système politique constituaient les deux plus importantes raisons qui avaient déterminé leur choix ${ }^{32}$. Face aux diverses défaillances identifiées en Irlande, comment les citoyens ont-ils réagi? Et quelles ont été en retour les réactions de l'État lui-même?

Appliquée à ces questions, l'analyse de l'économiste américain Albert O. Hirschman permet de remettre en perspective certaines des tensions qui traversent la société irlandaise ${ }^{33}$. Étudiant le comportement des individus face à la perception d'un déclin de la qualité d'un produit d'une entreprise (conception économique) ou de la qualité des biens collectifs offerts par la société qui n'offrent plus les bénéfices escomptés ou attendus (conception socio-politique), Albert O. Hirschman en concluait que l'expression du mécontentement pouvait prendre deux formes principales : «défection » ou "prise de parole».

\section{Défection ou l'émigration}

Particulièrement notable au $\mathrm{xx}^{\mathrm{e}}$ siècle en Irlande, la première forme d'expression représente une constante au regard des liens entre économie et émigration puisque de nombreux Irlandais ont quitté le pays pour tenter leur chance ailleurs, manifestant en grand nombre, à la fois leur mécontentement vis-à-vis de la situation existante ainsi que l'absence d'alternatives. Cette tendance a refait

regulators, bankers and property developers. " Brid Featherstone, "The Current Economic Crisis in Ireland: Why social work needs to be part of the challenge to a discredited system? ", Comunitania, janvier 2011.

30. Clodagh Harris, Gemma M. Carney et David M. Farrell, " Rule by the People? Alternative Perspectives on Citizen Participation in Democratic Policymaking ", Administration, vol. 60, no. 3, 2013, p. 201-209.

31. "The most obvious manifestation of our political problems is the sudden and very dramatic fall in public confidence in the government ". Niamh Hardiman, "The Impact of the Crisis on the Irish Political System ", op. cit., p. 3.

32. Michael Marsh et Kevin Cunningham, "A Positive Choice, or Anyone but Fianna Fáil? ", in M. Gallagher et M. Marsh, How Ireland Voted 2011, op. cit., p. 172-204, p. 187.

33. Albert O. Hirschman, Défection et prise de parole. Théorie et applications, trad. fr. par Claude Besseyrias, Exit, Voice and Loyalty: Responses to Decline in Firms, Organizations, and States [1970], Paris, Fayard, 1995. 
surface avec le ralentissement de la croissance, s'accentuant avec la crise alors que la décennie précédente avait davantage été marquée par un retour des Irlandais au pays et une forme de renouveau démographique avec l'arrivée de nouvelles populations. Les flux migratoires vers l'Irlande ont ainsi beaucoup diminué après 20072008, atteignant en 2010 leur niveau le plus bas depuis $1994^{34}$ (graphique 1).

Graphique 1 : Décomposition du solde migratoire en Irlande de 2001 à 2013 (en milliers)

(Source : D'après Central Statistics Office, Census of Population)

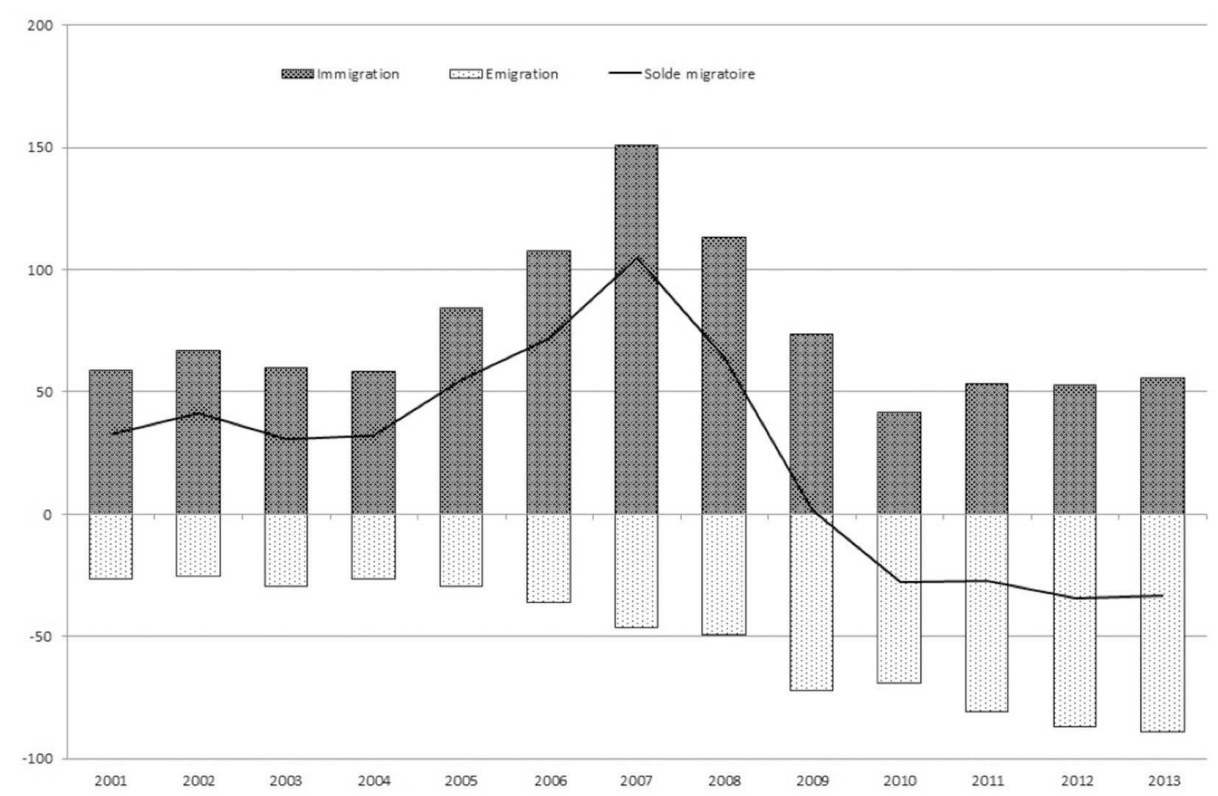

Depuis 2008, l'Irlande est redevenue un pays d'émigration. Celle-ci a augmenté de $16 \%$ entre 2010 et 2011, de $8 \%$ entre 2011 et 2012, puis de 2,2\% entre 2012 et 2013 . Mais, c'est surtout le détail des flux migratoires sortants qui est intéressant puisque l'analyse par nationalités montre que c'est chez les Irlandais qu'il y a la plus forte progression de l'émigration. Parmi les candidats à l'émigration entre avril 2012 et avril 2013, plus de la moitié étaient Irlandais (57,2 \%), soit une hausse de plus de 30 points entre 2008 et 2013 (tableau 1).

34. Mary Gilmartin, "The Changing landscape of Irish migration, 2000-2012 ", document de travail NISRA, $\mathrm{n}^{\circ} 69,2012$, p. 2. 
Tableau 1 : Flux migratoires sortants par nationalité entre 2008 et 2013 (en \%) (Source: D'après CSO, Population and Migration Estimates 2013, tableau 2)

\begin{tabular}{|c|c|c|c|c|c|c|}
\cline { 2 - 7 } \multicolumn{1}{c|}{} & 2008 & 2009 & 2010 & 2011 & $2012^{*}$ & $2013^{*}$ \\
\hline Irlandais & 26,6 & 26,7 & 41,8 & 52,1 & 53,4 & 57,2 \\
\hline Britanniques & 7,5 & 5,4 & 4,3 & 5,7 & 4,0 & 4,4 \\
\hline $\begin{array}{c}\text { Reste UE-15 } \\
\text { Soit Allemagne, Autriche, Belgique, } \\
\text { Danemark, Espagne, Finlande, France, } \\
\text { Grèce, Italie, Luxembourg, Pays-Bas, } \\
\text { Portugal et Suède. }\end{array}$ & 12,2 & 10,3 & 13,0 & 12,7 & 12,9 & 11,1 \\
\hline $\begin{array}{c}\text { UE-12 } \\
\text { Soit les dix pays entrés dans l'Union } \\
\text { européenne le 1 1er mai 2004 : Chypre, } \\
\text { Estonie, Hongrie, Lettonie, Lituanie, } \\
\text { Malte, Pologne, République Tchèque, } \\
\text { Slovaquie, Slovénie, ainsi que la Bulga- } \\
\text { rie et la Roumanie après le 1 1er janvier } \\
\text { 2007. }\end{array}$ & 35,0 & 42,4 & 27,5 & 17,2 & 17,0 & 15,7 \\
\hline Reste du monde & 18,3 & 15,3 & 13,4 & 12,3 & 12,7 & 11,6 \\
\hline
\end{tabular}

* Données provisoires en 2012 et 2013)

\section{Prise de parole, ou la manifestation pour faire bouger les choses}

La manifestation du mécontentement par la " prise de parole ", seconde forme d'expression définie par Hirschman, prend pour partie sa signification contemporaine dans la volonté de proposer des alternatives émanant d'individus ou de groupes d'individus pour répondre aux effets de la crise. Elle se manifeste également dans "l'organisation de la défiance ${ }^{35}$ " dont le développement accru, ces dernières années, provient en grande partie de la chute de confiance dans les institutions et groupes représentant la volonté populaire au sein des sociétés démocratiques.

Pour répondre à cette crise de confiance, la recherche d'une démocratie plus délibérative et participative a récemment fait surface en Irlande ${ }^{36}$. Ce changement de paradigme reflète ainsi un "appétit pour un renouveau démocratique ${ }^{37}$ " après deux décennies où une partie des acteurs de la société civile a pu prendre part aux discussions dans le cadre des accords tripartites, et après que le gouvernement a opté, en 2010, pour un programme d'austérité diminuant les salaires de la fonc-

35. Pierre Rosanvallon, La Contre-démocratie. La politique à l'âge de la défiance, Paris, Seuil, 2006, 12.

36. C. Harris, G. M. Carney et D. M. Farrell, "Rule by the People? ", op. cit., p. 202.

37. Gemma M. Carney et Clodagh Harris, (éds), Citizens'Voices : Experiments in Democratic Renewal and Reform, Political Studies Association of Ireland, 2012, disponible [www.psai.ie/specialist/PSAI-ebook-citizens-voices.pdf], 30 juin 2014, p. 1. 
tion publique et réduisant la protection sociale afin de baisser le déficit public et soutenir les banques ${ }^{38}$.

En rassemblant des organisations et des individus, deux mouvements, We the Citizens et Claiming Our Future, ont essayé de redonner un certain prestige au processus démocratique afin de relancer les débats sur les choix de société, notamment vis-à-vis du modèle économique, ainsi que sur les réponses à apporter à la crise et aux inégalités. Il y eut, dans ce sens, une certaine mobilisation citoyenne pour essayer de changer la société en regroupant des acteurs de la société civile engagés traditionnellement dans le quatrième pilier du partenariat social ${ }^{39}$. Mary P. Murphy a cependant montré que les réactions des Irlandais furent pour le moins mesurées, la société civile ne réagissant pas de la même manière qu'en Espagne ou en Grèce ${ }^{40}$. Toutefois, une étude très récente en Irlande a démontré que la pratique délibérative fonctionnait et que les citoyens irlandais pouvaient prendre des décisions éclairées lorsqu'ils avaient à la fois la possibilité de débattre et l'information nécessaire en leur possession ${ }^{41}$. Parallèlement, la volonté de réformer la sphère politique a été présente dans les programmes politiques des partis lors de l'élection de 2011, tous les partis appelant à plus d'engagement citoyen ${ }^{42}$.

"L'organisation de la défiance » évoquée plus haut n’est pas nouvelle en soi puisque les régimes démocratiques ont toujours cherché à mettre en place un ensemble de contre-pouvoirs, de pratiques et d'institutions destiné à compenser l'érosion de la confiance ${ }^{43}$. Cette défiance démocratique a alors comme objectif « de veiller à ce que le pouvoir élu reste fidèle à ses engagements, de trouver les moyens permettant de maintenir l'exigence initiale d'un service du bien commun ${ }^{44}$."

Trois modalités délimitent les contours de cette société de la défiance : les pouvoirs de surveillance, les pouvoirs de sanction et d'empêchement, ainsi que la montée en puissance du peuple-juge dans le cadre du déclin de réactivité des

38. Gemma M. Carney, Tony Dundon et Áine Ní Léime, "Protecting the Most Vulnerable” in an Economic Crisis: a Participatory Study of Civil Society Organisations in Ireland ", Voluntary Sector Review, vol. 3, no. 3, 2012, p. 329-346, p. 332.

39. Mary P. Murphy, "Participating in Popularizing Policy Alternatives: a case Study of Claiming our Future ", in Deiric Ó Broin et Mary P. Murphy, (éds.), Politics, Participation and Power. Civil Society and Public Policy in Ireland, Dublin, Glasnevin Publishing, 2013, p. 113-123.

40. Mary P. Murphy, "Civil Society in the Shadow of the Irish State ", Irish Journal of Sociology, vol. 19, no. 2, 2011, p. 170-187, p. 171, source accessible [http://eprints.nuim.ie/4589], consultée le 15 juin 2014.

41. David M. Farrell, Eoin O'Malley et Jane Suiter, "The 2011 We The Citizens Pilot Citizens' Assembly " in Gemma M. Carney et Clodagh Harris, (éd.), Citizens' Voices: Experiments in Democratic Renewal and Reform, Political Studies Association of Ireland, 2012, disponible [www.psai.ie/specialist/PSAI-ebook-citizens-voices.pdf], 30 juin 2014, p. 16-24, p. 23.

42. Ibid., p. 16.

43. Pierre Rosanvallon, La Contre-démocratie, op. cit., p. 12.

44. Ibid., p. 15. 
gouvernements face aux demandes des citoyens, soit un "peuple-surveillant ", un " peuple-véto » et un "peuple-juge ${ }^{45}$ ". La première dimension nous intéresse tout particulièrement ici et va de pair avec une demande croissante de voir les gouvernements rendre des comptes (principe d'accountability, difficilement traduisible en français), pratique d'autant plus prégnante que devient importante la notion de " transparence » dans le débat public. Elaine Byrne a ainsi montré que les vertus politiques et l'éthique de la responsabilité se sont métamorphosées au $\mathrm{xx}^{\mathrm{e}}$ siècle en Irlande, nécessitant sans doute encore davantage une vigilance citoyenne accrue due à cette lente érosion de la responsabilité et du service du bien commun auxquels doivent se plier les élus. L'essor d'Internet et des nouvelles technologies ces dernières décennies a rendu plus facile la surveillance des représentants élus, et l'exercice par chaque individu de ce pouvoir de surveillance ${ }^{46}$.

\section{Entre surveillance et volonté de transparence : retour sur deux exemples}

Deux exemples en Irlande seront succinctement considérés ${ }^{47}$. Le premier s'intéressera à une tentative visant à surveiller les actions de l'État et des représentants élus grâce à l'utilisation des nouvelles technologies ${ }^{48}$, tandis que le second présentera la manière dont l'Etat irlandais entend réagir aux demandes pour plus de transparence et d'accessibilité des données dans le cadre des politiques Open Data.

Le premier exemple est celui d'un site Internet dont le but est de surveiller l'action des parlementaires. Conçu en avril 2009 par John Handelaar et Gavin Sheridan ${ }^{49}$, le site KildareStreet.com, du nom de l'entrée principale du Parlement irlandais, reprend les recettes du site britannique theyworkforyou.com. Le site est conçu comme une plateforme d'échanges entre les élus qui expliquent, pour certains, les raisons de leur prise de position, et les questions que peuvent se poser les internautes. Il s'agit aussi de scruter un peu ce que font les parlementaires :

45. Ibid., p. 23.

46. Si ce pouvoir de surveillance n'est pas nouveau dans l'histoire des régimes démocratiques, Internet et les nouvelles technologies ont donné une impulsion nouvelle à cette activité de surveillance, laissant par la même occasion aux différents États la possibilité de surveiller encore plus leurs citoyens. En même temps, le progrès technologique offre aussi aux citoyens la possibilité de surveiller les actions des gouvernements, ce qui rend aujourd'hui très difficile de déterminer très précisément qui surveille qui.

47. La présentation est volontairement succincte et s'attache à proposer un instantané de la situation présente. Elle pourra être développée dans le cadre d'un prochain article.

48. Voir Dominique Cardon, La Démocratie Internet: Promesses et Limites, Paris, Seuil, 2010.

49. [http://www.eolasmagazine.iela-citizen-voice/], page consultée le 13 novembre 2013. 
kildarestreet.com est un site impartial dont l'objectif est de faciliter le contrôle par les citoyens de l'action de leurs représentants à la Chambre basse du Parlement irlandais ${ }^{50}$.

La position de l'État irlandais vis-à-vis de la transparence et du gouvernement ouvert suggère, pour le moment au moins, une posture plutôt ambivalente. Publié en octobre 2013 après quelques mois d'une consultation destinée à recueillir des éléments permettant à l'Irlande de pouvoir adhérer à l'Open Government Partnership ${ }^{51}$, le rapport dirigé par Nuala Haughey de Transparency International s'ouvrait sur la phrase suivante :

$[\mathrm{L}] \mathrm{a}$ participation citoyenne et "l'encapacitation ", auxquelles s'ajoutent la transparence et l'utilisation des nouvelles technologies, engendrent la responsabilisation ainsi que la croissance économique ${ }^{52}$.

La suite du rapport faisait état

d'un immense désir d'information de la part de la population, désir d'en connaître davantage sur la manière dont les ressources publiques sont allouées et sur la manière dont les institutions peuvent rendre des comptes aux citoyens ${ }^{53}$.

Si le processus semblait suivre son cours en 2013-2014 ${ }^{54}$, l'année précédant le lancement de l'initiative Open Government Partnership avait davantage montré un gouvernement irlandais semblant traîner des pieds ${ }^{55}$, ce malgré un Programme for

50. "KildareStreet.com is a non-partisan website which aims to make it easy for people to keep tabs on their elected representatives in the Houses of the Oireachtas. " [http://www. kildarestreet.com/], site consulté le 15 novembre 2013.

51. Open Government Partnership est une initiative lancée en 2011 par plusieurs pays avec l'objectif « d'offrir une plateforme internationale dans le cadre de réformes internes pour celles et ceux qui souhaitent rendre leur gouvernement plus ouverts, pouvant rendre des comptes et plus à l'écoute des citoyens ». Open Government Partnership, [http://www.opengovpartnership.org/], site consulté le 21 novembre 2013, " to provide an international platform for domestic reformers committed to making their governments more open, accountable, and responsive to citizens."

52. "[C]itizen participation and empowerment, supported by transparency and the use of new technology, generates accountability and economic growth. " Report of a Consultation with Civil Society Representatives and Citizens on Ireland's Participation in the Open Government Initiative, octobre 2013, p. 2, rapport consulté le 30 octobre 2013, [http://www.per.gov.ielopen-government-partnership-ogp/].

53. " [S] trong desire for information, how decisions are made, how public funds are spent, how individuals and institutions are held to account. " Report of a Consultation with Civil Society Representatives and Citizens on Ireland's Participation in the Open Government Initiative, op. cit., p. 2.

54. La manière dont les quatre piliers (Transparency, Accountability, Citizens' Participation, Technology and Innovation) s'articuleront dans la stratégie du gouvernement irlandais pourra apporter des réponses sur les choix politiques envisagés.

55. Le projet de rejoindre ce groupe de pays n'allait pas de soi en 2012, le gouvernement irlandais arguant du coût financier d'un tel projet, poussant certains observateurs comme D. Parfenov à dénoncer la prépondérance de l'ouverture économique sur l'ouverture politique. Lire Denis Parfenov, «Is Ireland closing the door to Open Government? ", [http://www.activecitizen.cclopen/tales_from_the_coalface/], 24 septembre 2012, page consultée le 14 novembre 2013. 
Government 2011-2016 élaboré conjointement par Fine Gael et Labour, les deux partis actuellement au pouvoir, qui soulignait les enjeux à venir et les solutions pour sortir le pays de la crise, insistant avant toute chose sur le besoin de faire des économies.

Le gouvernement s'attachera à refaire redémarrer l'économie, à rétablir la confiance, à corriger le système bancaire et soutiendra le maintien et la création d'emplois. [...] Parallèlement, il existe un besoin clairement identifié pour que notre système politique épouse le changement, partage le fardeau, et montre l'exemple. [...] Notre système politique, s'il souhaite regagner en crédibilité et pertinence, doit [...] changer. Et plus largement, notre système de gouvernement doit se moderniser, s'adapter à de nouvelles circonstances financières et commencer à produire de meilleurs services avec moins de ressources ${ }^{56}$.

Ce Programme commun promettait également que « l'ensemble du gouvernement " serait " transparent, rendrait des comptes et serait efficient ", et que la nouvelle équipe aurait la volonté de " mettre l'accent sur la responsabilisation pour les résultats à tous les niveaux de l'administration, du Ministre vers les agents, avec des conséquences notables, selon que ce soit un succès ou un échec ${ }^{57}$ ". Une autre ligne de ce programme indiquait que le gouvernement promettait une gouvernance ouverte partant du principe que « là où il existe du secret et une absence de responsabilité, il y a du gaspillage et de l'extravagance ${ }^{58} "$.

Un tel partenariat n’a cependant pas suscité un vif intérêt dans la presse irlandaise, ce qui peut être éventuellement mis sur le compte de sérieux débats en Irlande autour de la réforme du Freedom of Information Act. En effet, lors de la parution du rapport de N. Haughey, la presse irlandaise semblait plus insister sur la dimension économique que citoyenne d'une démarche d'Open Government en dépit des problèmes soulevés au cours des années précédentes.

Par ailleurs, il est intéressant de mesurer la position du ministre en charge du dossier. Ainsi, à l'issue du sommet sur la gouvernance ouverte de Londres fin octobre 2013, Brendan Howlin, ministre responsable des dépenses publiques et

56. "The Government will get our economy moving, restore confidence, fix our banking system and support the protection and creation of jobs. [...] In parallel, there is a clear need for our political system to embrace change, share the burden and lead by example. [...] Our political system, if it is to regain credibility and relevance, must change [...]. Wider than that, our system of Government must modernise, adapt to new financial circumstances and start to deliver better services with scarce resources. " Programme for Government 2011-2016, [http://www.taoiseach.gov.ieleng/ Work_Of_The_Department/Programme_for_Government/Programme_for_Government_2011-2016.pdf, p. 2-3.

57. " [T] he whole of Government to become more transparent, accountable and efficient. We will pin down accountability for results at every level of the public service from Ministers - down with clear consequences for success and failure. " Programme for Government 2011-2016, op. cit., p. 27-28.

58. "Where there is secrecy and unaccountability, there is waste and extravagance ", Programme for Government 2011-2016, op. cit., p. 27. 
de la réforme, annonçait des mesures dans le cadre de l'Open Data. L'idée était de donner une impulsion à l'Open Data en Irlande.

L'Open Data - réclamant plus d'ouverture dans l'accès aux données du secteur public - possède un potentiel fort pour apporter des avantages considérables au citoyen et à l'économie, impliquant en même temps un élargissement de l'ouverture, de la transparence et de la responsabilisation $^{59}$.

Malgré des engagements forts (signature de la charte du G8, mise en place d'un comité sur l'Open Data, d'un groupe de suivi de l'Open Data ainsi qu'un portail pour mettre à disposition les données du secteur public), le reste de l'intervention, rapporté sur le site du ministère, offrait ensuite une vision différente. La transparence semblait avoir disparu, tout comme la volonté de rendre les administrations comptables de leur action. Sur ce dernier point, le contrôle des dépenses et l'efficacité des administrations apparaissaient plus importants que le développement d'une culture de la transparence.

La formulation de ces engagements sur l'Open Data au Sommet peut devenir un moteur de l'innovation et la croissance économique, améliorer les services publics et renforcer la responsabilisation en créant une culture dans laquelle les données du secteur public sont largement accessibles pour la ré-utilisation et la production de bénéfices au profit de l'utilisateur ${ }^{60}$.

La dimension économique semblait ainsi revenir au premier plan puisqu'elle allait dans le sens des avantages économiques que peut offrir l'Open Data et sur lesquels insistent celles et ceux qui militent pour l'ouverture des données, notamment dans le monde anglo-saxon. Une telle perspective conduit néanmoins à l'affaiblissement des promesses démocratiques que ce mouvement d'ouverture des données porte également en lui.

À ce stade, deux observations peuvent être formulées. Si l'Irlande suit une tendance, visible dans d'autres pays européens, vers un retour à une démocratie plus participative et plus proche des préoccupations citoyennes, la route semble encore longue avant que le modèle économique d'hier devienne un modèle poli-

59. "Open Data - more open access to public sector data - has a significant potential to yield substantial benefits to the citizen and the economy as well as underpinning greater openness, transparency and accountability. " "Minister for Public Expenditure and Reform announcing major Open Data Initiative at Open Government Partnership summit in London ", [http://per.gov.ie/2013/10/31/minister-for-public-expenditure-and-reform-announcing-major-opendata-initiative-at-open-government-partnership-summit-in-london/], page consultée le 15 novembre 2013.

60. "The delivery of these commitments on Open Data at the Summit can drive innovation and economic growth, improve public services and strengthen accountability by creating a culture in which public sector data is widely accessible for re-use and the realisation of user benefits. "Ibid. 
tique. L'engagement citoyen via l'essor des nouvelles technologies ne rendra pas forcément la démocratie irlandaise plus vertueuse malgré les demandes pour plus de transparence et les engagements pris par les gouvernements au pouvoir. Enfin, il est encore difficile d'évaluer dans quelle mesure de tels engagements, visant à rendre plus transparent le fonctionnement de la puissance publique, ne cachent pas des desseins plus communicationnels. La recherche d'alternatives de société passera, à n'en point douter, par un savant mélange de ces diverses dimensions. 